\title{
Comparison of Trifecta Outcome Among Open, Laparoscopic, and Robotic-Assisted Partial Nephrectomy in Patients With Small Renal Masses: 10-Year Experience in Ramathibodi Hospital
}

Chaichant Soisrithong

Ramathibodi Hospital

Wit Viseshsindh

Ramathibodi Hospital

Wisoot Kongchareonsombat

Ramathibodi Hospital

Charoen Leenanupunth

Ramathibodi Hospital

Wachira Kochakarn

Ramathibodi Hospital

Pokket Sirisreetreerux

Ramathibodi Hospital

Premsant Sangkum

Ramathibodi Hospital

Kittinut Kijvikai

Ramathibodi Hospital

Pocharapong Jenjitranant ( $\nabla$ pocharapong@gmail.com )

Ramathibodi Hospital

Research Article

Keywords: partial nephrectomy, trifecta, Thai, laparoscopy, robotic-assisted partial nephrectomy

Posted Date: February 22nd, 2021

DOI: https://doi.org/10.21203/rs.3.rs-189966/v1

License: (c) (1) This work is licensed under a Creative Commons Attribution 4.0 International License.

Read Full License 


\section{Abstract}

Purpose: To compare the trifecta outcome and perioperative and postoperative outcomes among open partial nephrectomy (OPN), laparoscopic partial nephrectomy (LPN), and robotic-assisted partial nephrectomy (RAPN) in patients with small renal masses in Ramathibodi Hospital.

Methods: We retrospectively reviewed 141 patients who underwent partial nephrectomy from January 2009 to December 2018. The baseline characteristics and preoperative outcomes were compared among the three surgical approaches.

Results: Among the 141 patients, 42 (29.79\%), 29 (20.57\%), and 70 (49.64\%) patients underwent OPN, LPN, and RAPN, respectively. Among 116 patients with available data, 71 achieved the trifecta outcome [18 (56.25\%), 14 (56.00\%), and 39 (73.58\%) in the OPN, LPN, and RAPN group, respectively; $p=0.276$ ]. The rate of conversion to OPN was higher in the LPN than RAPN group. The number of patients who received intraoperative packed red cells was lowest in the RAPN group. The estimated blood loss was significantly lower in the LPN group than OPN and RAPN groups $(p=0.041)$. The operative time was shorter in the OPN group than LPN and RAPN groups $(p<0.001)$. Multivariate analysis showed that the intraoperative complication rate was a predictive factor for trifecta outcome achievement.

Conclusion: OPN had the shortest operative time. LPN had the highest rate of conversion to OPN and lowest estimated blood loss. The number of patients who received intraoperative packed red cells was lowest in the RAPN group. However, achievement of the trifecta outcome was not significantly different among the three groups. The predictive factor for trifecta outcome achievement was the intraoperative complication rate.

\section{Introduction}

Renal cell carcinoma (RCC) accounts for $2-3 \%$ of all adult neoplasms. Approximately 64,000 new cases are diagnosed each year in the United States, and 14,400 patients die every year. In contrast, the incidence and mortality rate are approximately 50\% lower in Asian-Americans/Pacific Islanders [1].

Renal masses may be either malignant or benign [2]. Small renal masses of $<4 \mathrm{~cm}$ are considered benign in $20-30 \%$ of cases [3]. Many clinical T1a cancers have an indolent biology [4]. The likelihood of malignancy increases with each 1-cm increase in diameter [5].

Common symptoms of renal masses are flank pain, a palpable mass, and hematuria. The incidental detection of renal masses [2] and the detection of small renal masses [6] have increased with improvements in imaging techniques [2].

Partial nephrectomy is being increasingly utilized for the treatment of renal masses [10]. The benefits of partial nephrectomy are improvement of morbidity and mortality, a decreased risk of development or 
progression of chronic kidney disease (CKD), and similarity of oncologic outcomes compared with radical nephrectomy [7-10].

Urologic complications, such as urine leakage or postoperative bleeding, are more common after partial than radical nephrectomy [11]. Computed tomography with/without contrast or magnetic resonance imaging with/without gadolinium is recommended for investigation of renal masses $[8,12]$. According to the 2017 American Urological Association guideline, partial nephrectomy is recommended for patients with CT1a renal masses and other conditions in which radical nephrectomy may compromise future renal function [8].

Partial nephrectomy is appropriate for patients with unilateral stage I to III tumors who have renal insufficiency, bilateral renal tumors, familial RCC, or a risk of progressive CKD because of young age or medical risk factors [12]. Open partial nephrectomy (OPN) was historically performed in all cases. However, laparoscopic partial nephrectomy (LPN) and robotic-assisted laparoscopic partial nephrectomy (RAPN) are now being increasing used [13]. The first robot-assisted laparoscopic prostatectomy was performed in 2000 [14], and robotic-assisted renal surgery was performed thereafter. RAPN has been performed in Ramathibodi Hospital since 2015.

The concept of the "trifecta" outcome has recently been used to evaluate surgical proficiency and includes negative surgical margins, no severe surgical complications, and $90 \%$ preservation of the estimated glomerular filtration rate (eGFR) postoperatively [15]. However, the factors that affect the trifecta outcome are unclear. This study was performed to compare the perioperative, postoperative, and trifecta outcomes of OPN, LPN, and RAPN and identify predictive factors for trifecta outcome achievement.

\section{Materials And Methods}

\section{Study population}

We retrospectively reviewed the medical records of all patients who underwent OPN, LPN, and RAPN at Ramathibodi Hospital from January 2009 to December 2018. Patients with incomplete data were excluded. Informed consents were obtained from all subjects or, if subjects are under 18 , from a parent and/or legal guardian.

The patients' baseline characteristics and disease- and treatment-related parameters were collected, including age, sex, body weight, height, body mass index, previous abdominal surgery, American Society of Anesthesiologists classification, underlying disease, size of renal mass, RENAL nephrometry score (radius, endophytic vs. exophytic, nearness to collecting system, anterior or posterior, and location relative to polar line), surgical approach (OPN, LPN, or RAPN), tumor pathology, and preoperative serum creatinine concentration. 
Perioperative and postoperative data were also collected, including the operative time (skin incision to skin closure time), estimated blood loss (EBL), blood transfusion, warm ischemia time, cold ischemia time, length of hospital stay (LOS), 1-year postoperative creatinine concentration and eGFR, and perioperative and postoperative complications. Trifecta outcome achievement was defined as the combination of negative surgical margins, no severe complications (Clavien-Dindo grade $0-2$ ), and a postoperative eGFR of $>90 \%$ of the preoperative eGFR. All methods were carried out in accordance with relevant guidelines and regulations

\section{Statistical analysis}

Predictive factors for trifecta outcome achievement were compared between patients who did and did not achieve the trifecta outcome using Student's t-test or the Mann-Whitney test for continuous variables and the chi-square test or Fisher's exact test for categorical variables. The patients' characteristics, perioperative and postoperative outcomes, and trifecta outcome achievement were compared among the three operative techniques using one-way analysis of variance or the Kruskal-Wallis test for continuous variables and the chi-square test for categorical variables. Univariate and multivariate analyses were performed using a logistic regression model to identify predictive factors associated with CKD, a risk of severe complications, and trifecta outcome achievement. All statistical analyses were performed with Stata v.14 (StataCorp, College Station, TX, USA). Statistical significance was defined as a p-value of < 0.05 .

\section{Results}

Among the 141 patients in this study, 42 (29.79\%) underwent OPN, 29 (20.57\%) underwent LPN, and 70 (49.64\%) underwent RAPN (Table 1). The patients' characteristics were similar among the three groups in terms of sex, age, body mass index, underlying disease, previous abdominal surgery, American Society of Anesthesiologists classification, and tumor size on imaging. Tumor complexity according to the RENAL nephrometry score was significantly greater in the RAPN than OPN and LPN groups $(p=0.005)$. 
Table 1

Patient characteristics according to type of operation

\begin{tabular}{|c|c|c|c|c|c|}
\hline \multirow[t]{2}{*}{ Patient characteristics } & \multirow{2}{*}{$\begin{array}{l}\text { Total } \\
(n=141)\end{array}$} & \multirow{2}{*}{$\begin{array}{l}\text { OPN } \\
(n=42)\end{array}$} & \multirow{2}{*}{$\begin{array}{l}\text { LPN } \\
(n=29)\end{array}$} & \multirow{2}{*}{$\begin{array}{l}\text { RAPN } \\
(n=70)\end{array}$} & \multirow{2}{*}{$\begin{array}{l}\mathrm{p}- \\
\text { value }\end{array}$} \\
\hline & & & & & \\
\hline \multicolumn{6}{|l|}{ Sex } \\
\hline Male & $68(48.23)$ & $18(42.86)$ & $10(34.48)$ & $40(57.14)$ & 0.086 \\
\hline Female & 73 (51.77) & $24(57.14)$ & $19(65.52)$ & $30(42.86)$ & \\
\hline Age, years & $\begin{array}{l}56.68 \pm \\
12.31\end{array}$ & $\begin{array}{l}56.09 \pm \\
13.22\end{array}$ & $58.55 \pm 10.17$ & $\begin{array}{l}56.28 \pm \\
12.65\end{array}$ & 0.658 \\
\hline $\mathrm{BMI}, \mathrm{kg} / \mathrm{m}^{2}$ & $25.40 \pm 4.28$ & $\begin{array}{l}25.38 \pm \\
4.35\end{array}$ & $25.73 \pm 4.55$ & $25.28 \pm 4.19$ & 0.895 \\
\hline DM & $26(18.44)$ & $9(21.43)$ & $7(24.14)$ & $10(14.29)$ & 0.432 \\
\hline HT & $76(53.90)$ & $22(52.38)$ & $15(51.72)$ & $39(55.71)$ & 0.911 \\
\hline DLP & $56(39.72)$ & $15(35.71)$ & $13(44.83)$ & $28(40.00)$ & 0.741 \\
\hline \multicolumn{6}{|l|}{ Chronic kidney disease } \\
\hline Stage 1 & $74(52.48)$ & $18(42.86)$ & $15(51.72)$ & $41(58.57)$ & 0.477 \\
\hline Stage 2 & $42(29.79)$ & $16(38.10)$ & $9(31.03)$ & $17(24.29)$ & \\
\hline Stage $3 a$ & $14(9.93)$ & $4(9.52)$ & $3(10.34)$ & $7(10.00)$ & \\
\hline Stage 3b & $7(4.96)$ & $3(7.14)$ & $2(6.90)$ & $2(2.86)$ & \\
\hline Stage 4 & $3(2.13)$ & $0(0.00)$ & $0(0.00)$ & $3(4.29)$ & \\
\hline Stage 5 & $1(0.71)$ & $1(2.38)$ & $0(0.00)$ & $0(0.00)$ & \\
\hline Single kidney & $10(7.09)$ & $4(9.52)$ & $1(3.45)$ & $5(7.14)$ & 0.618 \\
\hline \multicolumn{6}{|l|}{$\begin{array}{l}\text { Previous abdominal } \\
\text { surgery }\end{array}$} \\
\hline No & $86(60.99)$ & $26(61.90)$ & $19(65.52)$ & $41(58.57)$ & 0.804 \\
\hline Yes & $55(39.01)$ & $16(38.10)$ & $10(34.48)$ & $29(41.43)$ & \\
\hline \multicolumn{6}{|c|}{$\begin{array}{l}\text { Count previous abdominal } \\
\text { surgery }\end{array}$} \\
\hline I & $44(80.00)$ & $15(93.75)$ & $8(80.00)$ & $21(72.41)$ & 0.138 \\
\hline \multicolumn{6}{|c|}{ Data are presented as mean \pm standard deviation, $\mathrm{n}(\%)$, or median (interquartile range). } \\
\hline $\begin{array}{l}\text { OPN, open partial neph } \\
\text { nephrectomy; BMI, bod } \\
\text { American Society of Ar }\end{array}$ & $\begin{array}{l}\text {; LPN, lapar } \\
\text { index; DM, d } \\
\text { ologists; eGF }\end{array}$ & $\begin{array}{l}\text { pic partial r } \\
\text { tes mellitus } \\
\text { stimated gl }\end{array}$ & $\begin{array}{l}\text { arectomy; RAF } \\
\text {, hypertensio } \\
\text { erular filtration }\end{array}$ & $\begin{array}{l}\text { robot-assisted } \\
\text { LP, dyslipidem } \\
\text { te. }\end{array}$ & $\begin{array}{l}\text { artial } \\
; \text { ASA }\end{array}$ \\
\hline
\end{tabular}




\begin{tabular}{|c|c|c|c|c|c|}
\hline \multirow[t]{2}{*}{ Patient characteristics } & Total & OPN & LPN & RAPN & \multirow{2}{*}{$\begin{array}{l}\mathrm{p} \text { - } \\
\text { value }\end{array}$} \\
\hline & $(n=141)$ & $(n=42)$ & $(n=29)$ & $(n=70)$ & \\
\hline ॥ & $7(12.73)$ & $1(6.25)$ & $0(0.00)$ & $6(20.69)$ & \\
\hline III & $3(5.45)$ & $0(0.00)$ & $1(10.00)$ & $2(6.90)$ & \\
\hline IV & $1(1.82)$ & $0(0.00)$ & $1(10.00)$ & $0(0.00)$ & \\
\hline \multicolumn{6}{|l|}{ ASA class } \\
\hline Class I & $23(16.31)$ & $4(9.52)$ & $7(24.14)$ & $12(17.14)$ & 0.250 \\
\hline Class II & $55(39.01)$ & $14(33.33)$ & $9(31.03)$ & $32(45.71)$ & \\
\hline Class III & $60(42.55)$ & $22(52.38)$ & $13(44.83)$ & $25(35.71)$ & \\
\hline Class IV & $3(2.13)$ & $2(4.76)$ & $0(0.00)$ & $1(1.43)$ & \\
\hline \multicolumn{6}{|l|}{ Side } \\
\hline Left & $65(46.10)$ & $17(40.48)$ & $12(41.38)$ & $36(51.43)$ & 0.451 \\
\hline Right & $76(53.90)$ & $25(59.52)$ & $17(58.62)$ & $34(48.57)$ & \\
\hline Imaging size, mm & $3.2(2.3-4.8)$ & $\begin{array}{l}3.2(2.1- \\
6.0)\end{array}$ & $3.1(2.3-4.1)$ & $\begin{array}{l}3.4(2.3- \\
4.8)\end{array}$ & 0.983 \\
\hline \multicolumn{6}{|l|}{ RENAL score } \\
\hline Min-6 & $56(39.72)$ & $12(28.57)$ & $15(51.72)$ & $29(41.43)$ & 0.005 \\
\hline $7-9$ & $76(53.90)$ & $30(71.43)$ & $14(48.28)$ & $32(45.71)$ & \\
\hline $10-12$ & $9(6.38)$ & $0(0.00)$ & $0(0.00)$ & $9(12.86)$ & \\
\hline $\begin{array}{l}\text { Pathologic mass size, } \mathrm{cm} \\
(\mathrm{n}=134)\end{array}$ & $3(2-5)$ & $3(2.2-5.5)$ & $3(2.2-5.5)$ & $3(2-4.2)$ & 0.625 \\
\hline \multicolumn{6}{|l|}{ Pathology } \\
\hline Renal cell carcinoma & $70(49.65)$ & $18(42.86)$ & $11(37.93)$ & $41(58.57)$ & 0.204 \\
\hline Angiomyolipoma & $54(38.30)$ & $18(42.86)$ & $16(55.17)$ & $20(28.57)$ & \\
\hline Oncocytoma & $5(3.55)$ & $1(2.38)$ & $1(3.45)$ & $3(4.29)$ & \\
\hline Other & $12(8.51)$ & $5(11.90)$ & $1(3.45)$ & $6(8.57)$ & \\
\hline
\end{tabular}

Data are presented as mean \pm standard deviation, $\mathrm{n}$ (\%), or median (interquartile range).

OPN, open partial nephrectomy; LPN, laparoscopic partial nephrectomy; RAPN, robot-assisted partial nephrectomy; BMI, body mass index; DM, diabetes mellitus; HT, hypertension; DLP, dyslipidemia; ASA, American Society of Anesthesiologists; eGFR, estimated glomerular filtration rate. 


\begin{tabular}{|c|c|c|c|c|c|}
\hline \multirow[t]{2}{*}{ Patient characteristics } & Total & OPN & LPN & RAPN & \multirow{2}{*}{$\begin{array}{l}\mathrm{p}- \\
\text { value }\end{array}$} \\
\hline & $(n=141)$ & $(n=42)$ & $(n=29)$ & $(n=70)$ & \\
\hline \multicolumn{6}{|l|}{$\begin{array}{l}\text { Renal cell carcinoma }(n= \\
68)\end{array}$} \\
\hline Clear cell & $55(80.88)$ & $12(75.00)$ & 8 (72.73) & $35(85.37)$ & 0.146 \\
\hline Papillary & $6(8.82)$ & $1(6.25)$ & $1(9.09)$ & $4(9.76)$ & \\
\hline Chromophobe & $4(5.88)$ & $2(12.50)$ & $2(18.18)$ & $0(0.00)$ & \\
\hline Other & $3(4.41)$ & $1(6.25)$ & $0(0.00)$ & $2(4.88)$ & \\
\hline \multicolumn{6}{|l|}{ Preoperative eGFR } \\
\hline & $\begin{array}{l}90.7(70.9- \\
99.7)\end{array}$ & $\begin{array}{l}79.7(60.7- \\
97)\end{array}$ & $\begin{array}{l}86.9(72.0- \\
100.5)\end{array}$ & $\begin{array}{l}93.1(71.7- \\
103)\end{array}$ & 0.375 \\
\hline \multicolumn{6}{|l|}{$\begin{array}{l}\text { Chronic kidney disease ( } \mathrm{n} \\
=121 \text { ) }\end{array}$} \\
\hline No & $43(35.54)$ & $15(42.86)$ & $11(39.29)$ & $17(29.31)$ & 0.373 \\
\hline Yes & $78(64.46)$ & $20(57.14)$ & $17(60.71)$ & $41(70.69)$ & \\
\hline \multicolumn{6}{|c|}{ Data are presented as mean \pm standard deviation, $\mathrm{n}(\%)$, or median (interquartile range). } \\
\hline \multicolumn{6}{|c|}{$\begin{array}{l}\text { OPN, open partial nephrectomy; LPN, laparoscopic partial nephrectomy; RAPN, robot-assisted partial } \\
\text { nephrectomy; BMI, body mass index; DM, diabetes mellitus; HT, hypertension; DLP, dyslipidemia; ASA, } \\
\text { American Society of Anesthesiologists; eGFR, estimated glomerular filtration rate. }\end{array}$} \\
\hline
\end{tabular}

The perioperative and postoperative outcomes are shown in Table 2. The LOS was similar in the OPN, LPN, and RAPN groups ( 5 days for all). The rate of conversion to radical nephrectomy was also similar among the three groups. The rate of conversion to OPN was significantly lower in the RAPN than LPN group. EBL was significantly lower in the LPN group than in the OPN and RAPN groups. The warm ischemia time was similar in the LPN and RAPN groups. The operative time was significantly shorter in the OPN group than in the LPN and RAPN groups. The numbers of patients who received perioperative and postoperative packed red cells (PRC) were significantly higher in the OPN group than in the LPN and RAPN groups. 
Table 2

Perioperative and postoperative outcomes

\begin{tabular}{|c|c|c|c|c|c|}
\hline \multirow[t]{2}{*}{ Outcomes } & Total & OPN & LPN & RAPN & \multirow{2}{*}{$\begin{array}{l}\text { p- } \\
\text { value }\end{array}$} \\
\hline & $(n=141)$ & $(n=42)$ & $(n=29)$ & $(n=70)$ & \\
\hline Length of hospital stay, days & $5(4-6)$ & $5(4-6)$ & $5(4-7)$ & $5(4-6)$ & 0.451 \\
\hline \multicolumn{6}{|l|}{ Conversion to RN } \\
\hline No & $135(95.74)$ & $41(97.62)$ & $29(100)$ & $65(92.86)$ & 0.338 \\
\hline Yes & $6(4.26)$ & $1(2.38)$ & $0(0.00)$ & $5(7.14)$ & \\
\hline \multicolumn{6}{|l|}{ Conversion to open surgery } \\
\hline No & $139(98.58)$ & $42(100)$ & $27(93.10)$ & $70(100)$ & 0.041 \\
\hline Yes & $2(1.42)$ & $0(0.00)$ & $2(6.90)$ & $0(0.00)$ & \\
\hline Estimated blood loss, mL & $\begin{array}{l}300(150- \\
500)\end{array}$ & $\begin{array}{l}375(200- \\
700)\end{array}$ & $\begin{array}{l}200(100- \\
400)\end{array}$ & $\begin{array}{l}300(150- \\
500)\end{array}$ & 0.041 \\
\hline $\begin{array}{l}\text { Warm ischemia time, } \min (n= \\
134)\end{array}$ & $20(0-30)$ & & $27(20-31)$ & $25(20-34)$ & - \\
\hline $\begin{array}{l}\text { Cold ischemia time, } \min (n= \\
134)\end{array}$ & $25(0-35)$ & $25(0-35)$ & & & - \\
\hline Operative time, min & $\begin{array}{l}190(145- \\
235)\end{array}$ & $\begin{array}{l}125(95- \\
155)\end{array}$ & $\begin{array}{l}175(145- \\
200)\end{array}$ & $\begin{array}{l}220(190- \\
250)\end{array}$ & 0.000 \\
\hline \multicolumn{6}{|l|}{ Perioperative PRC } \\
\hline No & $117(82.98)$ & $29(69.05)$ & $25(86.21)$ & $63(90.00)$ & 0.015 \\
\hline Yes & $24(17.02)$ & $13(30.95)$ & $4(13.79)$ & $7(10.00)$ & \\
\hline PRC, mL & $\begin{array}{l}283(250- \\
544)\end{array}$ & $\begin{array}{l}267(246- \\
514)\end{array}$ & $\begin{array}{l}283(270- \\
542)\end{array}$ & $\begin{array}{l}288(249- \\
574)\end{array}$ & 0.763 \\
\hline \multicolumn{6}{|l|}{ PRC transfusion } \\
\hline No & $110(78.01)$ & 27 (64.29) & $24(82.76)$ & $59(84.29)$ & 0.037 \\
\hline Yes & $31(21.99)$ & $15(35.71)$ & $5(17.24)$ & $11(15.71)$ & \\
\hline PRC, mL & $\begin{array}{l}514(257- \\
783)\end{array}$ & $\begin{array}{l}307(246- \\
860)\end{array}$ & $\begin{array}{l}783(515- \\
864)\end{array}$ & $\begin{array}{l}448(270- \\
540)\end{array}$ & 0.394 \\
\hline Margin $(n=136)$ & & & & & \\
\hline
\end{tabular}

Data are presented as median (interquartile range) or $\mathrm{n}(\%)$.

OPN, open partial nephrectomy; LPN, laparoscopic partial nephrectomy; RAPN, robot-assisted partial nephrectomy; RN, radical nephrectomy; PRC, packed red cells; eGFR; estimated glomerular filtration rate. 


\begin{tabular}{|c|c|c|c|c|c|}
\hline \multirow[t]{2}{*}{ Outcomes } & Total & OPN & LPN & RAPN & \multirow{2}{*}{$\begin{array}{l}\mathrm{p} \text { - } \\
\text { value }\end{array}$} \\
\hline & $(n=141)$ & $(n=42)$ & $(n=29)$ & $(n=70)$ & \\
\hline Negative & $127(93.38)$ & $37(94.87)$ & $25(89.29)$ & $65(94.20)$ & 0.614 \\
\hline Positive & $9(6.62)$ & $2(5.13)$ & $3(10.71)$ & $4(5.80)$ & \\
\hline \multicolumn{6}{|l|}{ Intraoperative complications } \\
\hline No & $104(73.76)$ & 27 (64.29) & $21(72.41)$ & $56(80.00)$ & 0.184 \\
\hline Yes & $37(26.24)$ & $15(35.71)$ & $8(27.59)$ & $14(20.00)$ & \\
\hline $\begin{array}{l}\text { Penetration of } \\
\text { collecting system }\end{array}$ & $29(20.57)$ & $14(33.33)$ & $6(20.69)$ & $9(12.86)$ & \\
\hline $\begin{array}{l}\text { Conversion of } \\
\text { operation }\end{array}$ & $8(5.67)$ & $1(2.38)$ & $2(6.90)$ & $5(7.14)$ & \\
\hline \multicolumn{6}{|c|}{ Postoperative complications (Clavien-Dindo) } \\
\hline Grade $0-1$ & $106(75.18)$ & $33(78.57)$ & $23(79.31)$ & $50(71.43)$ & 0.922 \\
\hline Grade 2 & $29(20.57)$ & $6(14.29)$ & $6(20.69)$ & $17(24.29)$ & \\
\hline Grade 3a & $2(1.42)$ & $1(2.38)$ & $0(0.00)$ & $1(1.43)$ & \\
\hline Grade $4 a$ & $2(1.42)$ & $1(2.38)$ & $0(0.00)$ & $1(1.43)$ & \\
\hline Grade $4 b$ & $2(1.42)$ & $1(2.38)$ & $0(0.00)$ & $1(1.43)$ & \\
\hline Readmission & $3(2.14)$ & $1(2.44)$ & $0(0.00)$ & $2(2.86)$ & 0.999 \\
\hline \multicolumn{6}{|l|}{ eGFR } \\
\hline $1.0-1.5$ years & $\begin{array}{l}86.2(63.4- \\
95.1)\end{array}$ & $\begin{array}{l}76.2(55.5- \\
90.8)\end{array}$ & $\begin{array}{l}85.5(68.3- \\
96.0)\end{array}$ & $\begin{array}{l}87.9(69.8- \\
96.0)^{(6-8}\end{array}$ & 0.290 \\
\hline \multicolumn{6}{|c|}{ Data are presented as median (interquartile range) or $\mathrm{n}(\%)$. } \\
\hline \multicolumn{6}{|c|}{$\begin{array}{l}\text { OPN, open partial nephrectomy; LPN, laparoscopic partial nephrectomy; RAPN, robot-assisted partial } \\
\text { nephrectomy; RN, radical nephrectomy; PRC, packed red cells; eGFR; estimated glomerular filtration } \\
\text { rate. }\end{array}$} \\
\hline
\end{tabular}

The pathologic data in the OPN, LPN, and RAPN groups were similar in terms of the tumor size, negative margin rate, and rate of RCC, with no significant differences as shown in Table 3. 
Table 3

Trifecta outcome

\begin{tabular}{|c|c|c|c|c|c|}
\hline \multirow[t]{2}{*}{ Data } & Total & OPN & LPN & RAPN & \multirow[t]{2}{*}{ p-value } \\
\hline & $(n=141)$ & $(n=42)$ & $(n=29)$ & $(n=70)$ & \\
\hline \multicolumn{6}{|c|}{ Achieved eGFR at 1 year $(n=121)$} \\
\hline$<90 \%$ & $78(64.46)$ & $20(57.14)$ & $17(60.71)$ & $41(70.69)$ & 0.373 \\
\hline$\geq 90 \%$ & $43(35.54)$ & $15(42.86)$ & $11(39.29)$ & $17(29.31)$ & \\
\hline \multicolumn{6}{|l|}{ Margin $(n=136)$} \\
\hline Negative & $127(93.38)$ & $37(94.87)$ & $25(89.29)$ & $65(94.20)$ & 0.614 \\
\hline Positive & $9(6.62)$ & $2(5.13)$ & $3(10.71)$ & $4(5.80)$ & \\
\hline \multicolumn{6}{|c|}{ Severe complications (Clavien-Dindo grade $3-6$ ) } \\
\hline No & 135 (95.74) & $39(92.86)$ & $29(100)$ & $67(95.71)$ & 0.342 \\
\hline Yes & $6(4.26)$ & $3(7.14)$ & $0(0.00)$ & $3(4.29)$ & \\
\hline \multicolumn{6}{|c|}{ Trifecta outcome $(n=116)$} \\
\hline No & $45(38.79)$ & $14(43.75)$ & $13(48.15)$ & $18(31.58)$ & 0.276 \\
\hline Yes & $71(61.21)$ & $18(56.25)$ & $14(51.85)$ & $39(64.42)$ & \\
\hline \multicolumn{6}{|c|}{ Data are presented as $\mathrm{n}(\%)$. } \\
\hline
\end{tabular}

With respect to intraoperative complications, the incidence of collecting system perforation and severe complications (Clavien-Dindo grade 3-6) were similar among the three groups. The proportions of patients who achieved $\geq 90 \%$ preservation of the eGFR at 1 year postoperatively were also similar among the three groups, as shown in Table 4. 
Table 4

Numbers of patients who achieved trifecta outcome

\begin{tabular}{|llll|}
\hline & OPN & LPN & RAPN \\
\hline Achieved $\geq 90 \%$ eGFR at 1 year & $15 / 35(42.86)$ & $11 / 28(39.29)$ & $17 / 58(29.31)$ \\
\hline Negative margin & $37 / 39(94.87)$ & $25 / 28(89.29)$ & $65 / 69(94.2)$ \\
\hline No severe complications & $39 / 42(92.86)$ & $29 / 29(100)$ & $67 / 70(95.71)$ \\
\hline Achieved trifecta outcome & $18 / 32(56.25)$ & $14 / 27(51.85)$ & $39 / 57(64.42)$ \\
\hline Data are presented as n (\%). & & & \\
\hline $\begin{array}{l}\text { OPN, open partial nephrectomy; LPN, laparoscopic partial nephrectomy; RAPN, robot-assisted partial } \\
\text { nephrectomy; eGFR, estimated glomerular filtration rate }\end{array}$ & & \\
\hline
\end{tabular}

Because of incomplete data in 25 patients, 116 patients were included in the trifecta outcome analysis. The proportion of patients who achieved the trifecta outcome was similar among the three groups, as shown in Table 5. 
Table 5

Patient characteristics according to achievement of trifecta outcome

\begin{tabular}{|c|c|c|c|c|}
\hline \multirow[t]{2}{*}{ Patient characteristics } & \multirow{2}{*}{$\begin{array}{l}\text { Total } \\
(n=116)\end{array}$} & \multirow{2}{*}{$\begin{array}{l}\text { Non-Trifecta } \\
(n=45)\end{array}$} & \multirow{2}{*}{$\begin{array}{l}\text { Trifecta } \\
(n=71)\end{array}$} & \multirow{2}{*}{$\begin{array}{l}\mathrm{p}- \\
\text { value }\end{array}$} \\
\hline & & & & \\
\hline \multicolumn{5}{|l|}{ Sex } \\
\hline Male & $60(51.72)$ & $30(66.67)$ & $30(42.25)$ & 0.010 \\
\hline Female & $56(48.28)$ & 15 (33.33) & $41(57.75)$ & \\
\hline Age, years & $57.40 \pm 11.61$ & $57.93 \pm 12.67$ & $57.07 \pm 10.96$ & 0.698 \\
\hline $\mathrm{BMI}, \mathrm{kg} / \mathrm{m}^{2}$ & $25.16 \pm 4.27$ & $25.81 \pm 3.97$ & $24.75 \pm 4.44$ & 0.196 \\
\hline DM & $20(17.24)$ & $11(24.44)$ & $9(12.68)$ & 0.102 \\
\hline HT & $61(52.59)$ & $24(53.33)$ & $37(52.11)$ & 0.898 \\
\hline DLP & $44(37.93)$ & 17 (37.78) & $27(38.03)$ & 0.978 \\
\hline \multicolumn{5}{|l|}{ Chronic kidney disease } \\
\hline Stage 1 & $62(53.45)$ & $18(40.00)$ & $44(61.97)$ & 0.088 \\
\hline Stage 2 & $35(30.17)$ & 17 (37.78) & $18(25.35)$ & \\
\hline Stage 3a & $11(9.48)$ & $7(15.56)$ & $4(5.63)$ & \\
\hline Stage 3b & $6(5.17)$ & $3(6.67)$ & $3(4.23)$ & \\
\hline Stage 4 & $1(0.86)$ & $0(0.00)$ & $1(1.41)$ & \\
\hline Stage 5 & $1(0.86)$ & $0(0.00)$ & $1(1.41)$ & \\
\hline Single kidney & $7(6.03)$ & $1(2.22)$ & $6(8.45)$ & 0.245 \\
\hline \multicolumn{5}{|c|}{ Previous abdominal surgery } \\
\hline No & $72(62.07)$ & $29(64.44)$ & $43(60.56)$ & 0.675 \\
\hline Yes & $44(37.93)$ & $16(35.56)$ & $28(39.44)$ & \\
\hline \multicolumn{5}{|c|}{$\begin{array}{l}\text { Count previous abdominal } \\
\text { surgery }\end{array}$} \\
\hline I & 35 (79.55) & $11(68.75)$ & $24(85.71)$ & 0.390 \\
\hline
\end{tabular}

Data are presented as $\mathrm{n}(\%)$, mean \pm standard deviation, or median (interquartile range).

BMI, body mass index; DM, diabetes mellitus; HT, hypertension; DLP, dyslipidemia; ASA, American Society of Anesthesiologists; PRC, packed red cells; eGFR, estimated glomerular filtration rate; OPN, open partial nephrectomy; LPN, laparoscopic partial nephrectomy; RAPN; robot-assisted partial nephrectomy. 


\begin{tabular}{|c|c|c|c|c|}
\hline \multirow[t]{2}{*}{ Patient characteristics } & \multirow{2}{*}{$\begin{array}{l}\text { Total } \\
(n=116)\end{array}$} & \multirow{2}{*}{$\begin{array}{l}\text { Non-Trifecta } \\
(n=45)\end{array}$} & \multirow{2}{*}{$\begin{array}{l}\text { Trifecta } \\
(n=71)\end{array}$} & \multirow{2}{*}{$\begin{array}{l}p- \\
\text { value }\end{array}$} \\
\hline & & & & \\
\hline ॥ & $6(13.64)$ & $3(18.75)$ & $3(10.71)$ & \\
\hline III & $2(4.55)$ & $1(6.25)$ & $1(3.57)$ & \\
\hline IV & $1(2.27)$ & $1(6.25)$ & $0(0.00)$ & \\
\hline \multicolumn{5}{|l|}{ ASA class } \\
\hline Class I & $22(18.97)$ & $5(11.11)$ & $17(23.94)$ & 0.105 \\
\hline Class II & $44(37.93)$ & $17(37.78)$ & $27(38.03)$ & \\
\hline Class III & $48(41.38)$ & $21(46.67)$ & 27 (38.03) & \\
\hline Class IV & $2(1.72)$ & $2(4.44)$ & $0(0.00)$ & \\
\hline \multicolumn{5}{|l|}{ Side } \\
\hline Left & $50(43.10)$ & $19(42.22)$ & $31(43.66)$ & 0.879 \\
\hline Right & $66(56.90)$ & $26(57.78)$ & $40(56.34)$ & \\
\hline Size, mm & $3.2(2.3-4.8)$ & $3.2(2.2-4.8)$ & $3.4(2.6-4.8)$ & 0.182 \\
\hline RENAL score & $7(6-8)$ & $7(6-8)$ & $8(7-10)$ & 0.324 \\
\hline \multicolumn{5}{|l|}{ RENAL score group } \\
\hline Min-6 & $45(38.79)$ & $17(37.78)$ & $28(39.44)$ & 0.641 \\
\hline $7-9$ & $66(56.90)$ & $25(55.56)$ & $41(57.75)$ & \\
\hline $10-12$ & $5(4.31)$ & $3(6.67)$ & $2(2.82)$ & \\
\hline Length of hospital stay, days & $5(4-6)$ & $5(4-6)$ & $9(4-11)$ & 0.000 \\
\hline $\begin{array}{l}\text { Warm ischemia time, } \min (n= \\
111)\end{array}$ & $20(0-30)$ & $20(0-30)$ & $25(10-30)$ & 0.905 \\
\hline $\begin{array}{l}\text { Cold ischemia time, } \min (\mathrm{n}= \\
111)\end{array}$ & $0(0-0)$ & $0(0-0)$ & $0(0-17.5)$ & 0.109 \\
\hline Operative time, min & $190(145-235)$ & $185(145-230)$ & $242(185-285)$ & 0.321 \\
\hline
\end{tabular}

Data are presented as $\mathrm{n}(\%)$, mean \pm standard deviation, or median (interquartile range).

BMI, body mass index; DM, diabetes mellitus; HT, hypertension; DLP, dyslipidemia; ASA, American Society of Anesthesiologists; PRC, packed red cells; eGFR, estimated glomerular filtration rate; OPN, open partial nephrectomy; LPN, laparoscopic partial nephrectomy; RAPN; robot-assisted partial nephrectomy. 


\begin{tabular}{|c|c|c|c|c|}
\hline \multirow[t]{2}{*}{ Patient characteristics } & Total & Non-Trifecta & Trifecta & \multirow{2}{*}{$\begin{array}{l}\mathrm{p} \text { - } \\
\text { value }\end{array}$} \\
\hline & $(n=116)$ & $(n=45)$ & $(n=71)$ & \\
\hline No & $97(83.62)$ & $36(80.00)$ & $61(85.92)$ & 0.402 \\
\hline Yes & $19(16.38)$ & $9(20.00)$ & $10(14.08)$ & \\
\hline PRC, mL & $283(252-448)$ & $284(256-594)$ & $274(243-307)$ & 0.327 \\
\hline \multicolumn{5}{|l|}{ PRC transfusion } \\
\hline No & $92(79.31)$ & $34(75.56)$ & $58(81.69)$ & 0.427 \\
\hline Yes & $24(20.69)$ & $11(24.44)$ & $13(18.31)$ & \\
\hline $\mathrm{PRC}, \mathrm{mL}$ & $377(258-687)$ & $780(256-864)$ & $282(260-448)$ & 0.034 \\
\hline $\begin{array}{l}\text { Pathologic mass size, } \mathrm{cm}(\mathrm{n}= \\
\text { 113) }\end{array}$ & $3(2-5)$ & $3(2-5)$ & $3.8(2.6-5.0)$ & 0.261 \\
\hline \multicolumn{5}{|l|}{ Margin } \\
\hline Negative & $109(93.97)$ & $38(84.44)$ & $71(100)$ & 0.001 \\
\hline Positive & $7(6.03)$ & $7(15.56)$ & $0(0.00)$ & \\
\hline \multicolumn{5}{|l|}{ Pathology } \\
\hline Renal cell carcinoma & $59(50.86)$ & $23(51.11)$ & $36(50.70)$ & 0.700 \\
\hline Angiomyolipoma & $43(37.07)$ & $15(33.33)$ & $28(39.44)$ & \\
\hline Oncocytoma & $5(4.31)$ & $3(6.67)$ & $2(2.82)$ & \\
\hline Other & $9(7.76)$ & $4(8.89)$ & $5(7.04)$ & \\
\hline \multicolumn{5}{|l|}{ Renal cell carcinoma $(n=68)$} \\
\hline Clear cell & 46 (77.97) & $16(69.57)$ & $30(83.33)$ & 0.623 \\
\hline Papillary & $6(10.17)$ & $3(13.04)$ & $3(8.33)$ & \\
\hline Chromophobe & $4(6.78)$ & $2(8.70)$ & $2(5.56)$ & \\
\hline Other & $3(5.08)$ & $2(8.70)$ & $1(2.78)$ & \\
\hline \multicolumn{5}{|l|}{ Intraoperative complications } \\
\hline No & $89(76.72)$ & $28(62.22)$ & $61(85.92)$ & 0.003 \\
\hline
\end{tabular}

Data are presented as $\mathrm{n}(\%)$, mean \pm standard deviation, or median (interquartile range).

BMI, body mass index; DM, diabetes mellitus; $H T$, hypertension; DLP, dyslipidemia; ASA, American Society of Anesthesiologists; PRC, packed red cells; eGFR, estimated glomerular filtration rate; OPN, open partial nephrectomy; LPN, laparoscopic partial nephrectomy; RAPN; robot-assisted partial nephrectomy. 


\begin{tabular}{|c|c|c|c|c|}
\hline \multirow[t]{2}{*}{ Patient characteristics } & \multirow{2}{*}{$\begin{array}{l}\text { Total } \\
(n=116)\end{array}$} & \multirow{2}{*}{$\begin{array}{l}\text { Non-Trifecta } \\
(n=45)\end{array}$} & \multirow{2}{*}{$\begin{array}{l}\text { Trifecta } \\
(n=71)\end{array}$} & \multirow{2}{*}{$\begin{array}{l}\mathrm{p}- \\
\text { value }\end{array}$} \\
\hline & & & & \\
\hline Yes & $27(23.28)$ & $17(37.78)$ & $10(14.08)$ & \\
\hline \multicolumn{5}{|l|}{ Creatinine } \\
\hline Preoperative & $\begin{array}{l}0.87(0.68- \\
1.09)\end{array}$ & $0.86(0.67-1.06)$ & $\begin{array}{l}1.60(1.48- \\
2.50)\end{array}$ & 0.010 \\
\hline 2-4 weeks & $\begin{array}{l}0.97(0.71- \\
1.22)\end{array}$ & $0.95(0.70-1.19)$ & $\begin{array}{l}1.99(1.52- \\
2.10)\end{array}$ & 0.003 \\
\hline \multicolumn{5}{|l|}{ eGFR } \\
\hline Preoperative & $\begin{array}{l}90.7(70.9- \\
99.7)\end{array}$ & $\begin{array}{l}92.3(72.0- \\
100.6)\end{array}$ & $\begin{array}{l}36.3(28.0- \\
53.5)\end{array}$ & 0.046 \\
\hline 2-4 weeks & $\begin{array}{l}77.9(58.5- \\
94.9)\end{array}$ & $78.4(60.3-96.0)$ & $\begin{array}{l}28.0(22.0- \\
52.7)\end{array}$ & 0.017 \\
\hline $1-3$ months & $\begin{array}{l}86.8(63.1- \\
97.7)\end{array}$ & $87.2(64.6-98.2)$ & $\begin{array}{l}43.1(18.1- \\
50.6)\end{array}$ & 0.003 \\
\hline \multicolumn{5}{|l|}{ Operation type } \\
\hline OPN & $32(27.59)$ & $14(31.11)$ & $18(25.35)$ & 0.276 \\
\hline LPN & $27(23.28)$ & $13(28.89)$ & $14(19.72)$ & \\
\hline RAPN & $57(49.14)$ & $18(40.00)$ & $39(54.93)$ & \\
\hline \multicolumn{5}{|c|}{ Data are presented as $n(\%)$, mean \pm standard deviation, or median (interquartile range). } \\
\hline \multicolumn{5}{|c|}{$\begin{array}{l}\text { BMI, body mass index; DM, diabetes mellitus; } \mathrm{HT} \text {, hypertension; DLP, dyslipidemia; ASA, American } \\
\text { Society of Anesthesiologists; PRC, packed red cells; eGFR, estimated glomerular filtration rate; OPN, } \\
\text { open partial nephrectomy; LPN, laparoscopic partial nephrectomy; RAPN; robot-assisted partial } \\
\text { nephrectomy. }\end{array}$} \\
\hline
\end{tabular}

In the univariate analysis, the factors that affected the trifecta outcome were male sex, LOS, rate of intraoperative complications, and rate of postoperative complications. In the multivariate analysis, the factors that affected the trifecta outcome were the LOS and rate of intraoperative complications, as shown in Table 6. 
Table 6

Predictive factors for achievement of trifecta outcome

\begin{tabular}{|c|c|c|c|}
\hline \multirow[t]{2}{*}{ Predictive factors } & Univariate & \multirow[t]{2}{*}{ p-value } & Multivariate \\
\hline & OR $(95 \% \mathrm{Cl})$ & & OR $(95 \% \mathrm{Cl})$ \\
\hline
\end{tabular}

Sex

Male

Female

Age (years)

BMI $\left(\mathrm{kg} / \mathrm{m}^{2}\right)$

DM

HT

DLP

Chronic kidney disease

Stage 1

Stage 2

Stage 3a

Stage $3 b$

Stage 4

Stage 5

Single kidney

Previous abdominal surgery

No

Yes

Count previous abdominal surgery

I

II

III
1

$2.733(1.25-5.95) \quad 0.011$

$0.993(0.96-1.02) \quad 0.696$

$0.943(0.86-1.03) \quad 0.197$

$0.448(0.16-1.18) \quad 0.107$

$0.952(0.45-2.01) \quad 0.898$

$1.011(0.46-2.18) \quad 0.978$
1

$0.433(0.18-1.02) \quad 0.057$

$0.233(0.06-0.89) \quad 0.034$

$0.409(0.07-2.22) \quad 0.300$

$$
4.061(0.47-34.91) \quad 0.202
$$

1

$1.180(0.54-2.55) \quad 0.675$

$\mathrm{OR}$, odds ratio; $\mathrm{Cl}$, confidence interval; $\mathrm{BMI}$, body mass index; $\mathrm{DM}$, diabetes mellitus; $\mathrm{HT}$, hypertension; DLP, dyslipidemia; ASA, American Society of Anesthesiologists; PRC, packed red cells; eGFR, estimated glomerular filtration rate; OPN, open partial nephrectomy; LPN, laparoscopic partial nephrectomy; RAPN; robot-assisted partial nephrectomy. 


\begin{tabular}{|c|c|c|c|c|}
\hline \multirow[t]{2}{*}{ Predictive factors } & Univariate & \multirow[t]{2}{*}{ p-value } & Multivariate & \multirow[t]{2}{*}{ p-value } \\
\hline & OR $(95 \% \mathrm{Cl})$ & & OR $(95 \% \mathrm{Cl})$ & \\
\hline IV & - & - & & \\
\hline \multicolumn{5}{|l|}{ ASA class } \\
\hline Class I & 1 & & & \\
\hline Class II & $0.467(0.14-1.50)$ & 0.201 & & \\
\hline Class III & $0.378(0.12-1.19)$ & 0.097 & & \\
\hline Class IV & - & & & \\
\hline \multicolumn{5}{|l|}{ Side } \\
\hline Left & 1 & & & \\
\hline Right & $0.942(0.44-2.01)$ & 0.879 & & \\
\hline Size (mm) & $0.899(0.77-1.04)$ & 0.167 & & \\
\hline RENAL score & $0.858(0.68-1.08)$ & 0.198 & & \\
\hline \multicolumn{5}{|l|}{ RENAL score group } \\
\hline Min-6 & 1 & & & \\
\hline $7-9$ & $0.995(0.45-2.17)$ & 0.991 & & \\
\hline $10-12$ & $0.404(0.06-2.67)$ & 0.348 & & \\
\hline Length of hospital stay (days) & $0.638(0.50-0.81)$ & 0.000 & $0.644(0.49-0.83)$ & 0.001 \\
\hline Warm ischemia time (min) & $0.992(0.96-1.02)$ & 0.537 & & \\
\hline Cold ischemia time (min) & $0.973(0.94-1.00)$ & 0.067 & & \\
\hline Operative time (min) & $0.996(0.99-1.00)$ & 0.196 & & \\
\hline Pathologic mass size (cm) & $0.938(0.82-1.06)$ & 0.320 & & \\
\hline \multicolumn{5}{|l|}{ Pathology } \\
\hline Renal cell carcinoma & 1 & & & \\
\hline Angiomyolipoma & $1.192(0.52-2.69)$ & 0.673 & & \\
\hline Oncocytoma & $0.425(0.06-2.74)$ & 0.370 & & \\
\hline
\end{tabular}

$\mathrm{OR}$, odds ratio; $\mathrm{Cl}$, confidence interval; $\mathrm{BMI}$, body mass index; $\mathrm{DM}$, diabetes mellitus; $\mathrm{HT}$, hypertension; DLP, dyslipidemia; ASA, American Society of Anesthesiologists; PRC, packed red cells; eGFR, estimated glomerular filtration rate; OPN, open partial nephrectomy; LPN, laparoscopic partial nephrectomy; RAPN; robot-assisted partial nephrectomy. 


\begin{tabular}{|c|c|c|c|c|}
\hline \multirow[t]{2}{*}{ Predictive factors } & Univariate & \multirow[t]{2}{*}{ p-value } & Multivariate & \multirow[t]{2}{*}{ p-value } \\
\hline & OR (95\%Cl) & & OR $(95 \% \mathrm{Cl})$ & \\
\hline Other & $0.798(0.19-3.28)$ & 0.755 & & \\
\hline
\end{tabular}

Renal cell carcinoma

\begin{tabular}{lll} 
Clear cell & 1 & \\
\hline Papillary & $0.533(0.09-2.95)$ & 0.472 \\
\hline Chromophobe & $0.533(0.06-4.15)$ & 0.548 \\
\hline Other & $0.267(0.02-3.17)$ & 0.295
\end{tabular}

Intraoperative complications

$\begin{array}{lllll}\text { No } & 1 & & 1 & \\ \text { Yes } & 0.270(0.11-0.66) & 0.004 & 0.328(0.12-0.89) & 0.029\end{array}$

Creatinine

\begin{tabular}{lll} 
Preoperative & $0.980(0.64-1.49)$ & 0.926 \\
\hline $2-4$ weeks & $0.123(0.03-0.53)$ & 0.005
\end{tabular}

eGFR

\begin{tabular}{lll} 
Preoperative & $1.011(0.99-1.03)$ & 0.188 \\
\hline $2-4$ weeks & $1.026(1.00-1.05)$ & 0.018 \\
\hline $1-3$ months & $1.028(1.01-1.05)$ & 0.007
\end{tabular}

Operation type

\begin{tabular}{lll} 
OPN & 1 & \\
\hline LPN & $0.837(0.29-2.34)$ & 0.736 \\
\hline RAPN & $1.685(0.68-4.12)$ & 0.253
\end{tabular}

$\mathrm{OR}$, odds ratio; $\mathrm{Cl}$, confidence interval; $\mathrm{BMI}$, body mass index; $\mathrm{DM}$, diabetes mellitus; $\mathrm{HT}$, hypertension; DLP, dyslipidemia; ASA, American Society of Anesthesiologists; PRC, packed red cells; eGFR, estimated glomerular filtration rate; OPN, open partial nephrectomy; LPN, laparoscopic partial nephrectomy; RAPN; robot-assisted partial nephrectomy.

\section{Discussion}

Partial nephrectomy is an appropriate operation for the treatment of renal masses. In the past, OPN was the standard technique. LPN was later developed and is now increasing in use. In Ramathibodi Hospital, RAPN was first performed in 2015. According to the 2017 American Urological Association guideline [8] 
and the 12th edition of Campbell Walsh Wein Urology [2], partial nephrectomy is recommended for patients with a cT1a renal mass, solitary kidney, bilateral renal tumors, familial RCC, and CKD or proteinuria as well as young patients with multifocal masses or comorbidities that may impact renal function in the future. The most common complications after partial nephrectomy are urine leakage, postoperative bleeding, urinary tract infection, arteriovenous malformations, pseudoaneurysms, and renal abscesses [11].

In the present study, the three groups had similar baseline patient and tumor characteristics except for the RENAL nephrometry score, which was highest in the RAPN group $(p=0.005)$. The EBL was highest in the OPN group (OPN $>$ RAPN $>$ LPN, $p=0.041$ ). The percentage of patients who required perioperative PRC transfusion (OPN $>$ LPN $>$ RAPN, $p=0.015)$ and postoperative PRC transfusion $(p=0.037)$ was lowest in the RAPN group. The operative time was longest in the RAPN group (RAPN > LPN > OPN, $p<0.001$ ). LOS was not different among the three groups. The rate of positive surgical margins was not significantly different among the three groups but was highest in the LPN group. The rate of collecting system penetration was lowest in the RAPN group. No injury to major vessels or other abdominal organs occurred in any group.

Six patients required conversion to radical nephrectomy. One patient who underwent OPN was converted to open radical nephrectomy because of massive bleeding $(E B L$ of $4300 \mathrm{~mL})$ after unclamping of the renal artery (RENAL score of $8 \mathrm{~A}$ ). The other five patients who underwent RAPN were converted to roboticassisted radical nephrectomy because of high mass complexity in four patients (RENAL scores of 10P, 10P, 11A, and 10P) and mass involvement of the renal hilum in one patient (RENAL score of 6P).

Two patients underwent conversion to open surgery (LPN to OPN) because of massive adhesion and no progression of LPN in one patient and massive bleeding (EBL of $1500 \mathrm{~mL}$ ) after unclamping of the renal artery in the other patient.

Three patients developed severe postoperative complications after OPN. The first patient had a grade 3a postoperative arteriovenous fistula (AVF) $s / p$ angiogram requiring embolization. The second patient had grade 4 postoperative respiratory failure requiring mechanical ventilation. The third patient had grade $4 \mathrm{~b}$ postoperative cardiac arrest with atrial fibrillation requiring rapid ventricular response, respiratory failure with hospital-acquired pneumonia requiring mechanical ventilation, and acute kidney injury requiring hemodialysis.

Three patients developed severe postoperative complications after RAPN. The first patient had a grade 3a postoperative AVF s/p angiogram requiring embolization. The second patient had grade $4 a$ postoperative end-stage renal disease requiring long-term hemodialysis. The third patient had grade $4 \mathrm{~b}$ postoperative respiratory failure requiring mechanical ventilation and end-stage renal disease requiring long-term renal replacement therapy.

The number of patients with $\geq 90 \%$ preservation of the eGFR at the 1-year follow-up was not significantly different among the three groups. The proportion of patients $(n=116)$ who achieved the trifecta outcome 
was also similar among the groups $(p=0.736)$. In the multivariate analysis, the factors that affected the trifecta outcome were the LOS and rate of intraoperative complications.

Three patients were readmitted. One patient was given intravenous antibiotics to treat an infected intraabdominal collection after RAPN, one underwent angiography and embolization of a renal AVF after RAPN, and one underwent angiography and embolization of a renal AVF after OPN.

As in our study, Mehra et al. [16] found that blood loss was lowest in the RAPN group (OPN $>$ LPN $>$ RAPN, $p=0.042$ ). This can be explained by better visualization of the anatomy and movement control of instruments in RAPN. Similarly, Khalifeh et al. [17] concluded that the rate of a positive surgical margin was lower in RAPN than LPN. This may be due to technical difficulty when performing LPN, especially when the mass is in the upper pole or a posterior location.

Both Zargar et al. [18] and Xia et al. [19] reported that the rate of intraoperative complications was similar in OPN and RAPN. According to Khalifeh et al. [17] and Porpiglia et al. [13], the rate of postoperative complications was lower in RAPN than in LPN and OPN.

As in our study, Zargar et al. [20] found that the postoperative creatinine concentration and eGFR were not different between LPN and RAPN. Yerram et al. [15] found that the proportion of patients with $\geq 90 \%$ eGFR preservation at follow-up was not different between OPN and RAPN.

As in our study, Yerram et al. [15] found that achievement of the trifecta outcome (defined as negative surgical margins, no urologic complications, and $\geq 90 \%$ eGFR preservation at last follow-up) was not different between OPN and RAPN. This may be explained by the fact that LPN is inferior to both surgical OPN and RAPN because of its more challenging surgical techniques.

Zargar et al. [20] compared 646 LPN with 1185 RAPN procedures and defined the trifecta outcome as negative surgical margins, no perioperative complications, and a warm ischemia time of $\leq 25 \mathrm{~min}$. They found that the factors predicting achievement of the trifecta outcome were the type of surgery (RAPN), tumor size, EBL, and operative time.

As in our study, Mehra et al. [16] found that the operative time was not different among the three groups. In contrast, however, Khalifeh et al. [17] found that the operative time was shorter for RAPN than LPN. This can be explained by the steep learning curve of RAPN and the greater complexity of renal masses (higher RENAL scores) in the RAPN group in our study.

In contrast to our study, Han et al. [21] and Kim et al. [22] concluded that the LOS was significantly shorter for RAPN than OPN. LOS can be affected by many factors, including patient pain control, patient preference, socioeconomic status, and our institute practice.

Khalifeh et al. [17] and Porpiglia et al. [13] concluded that the rate of postoperative complications was lower in RAPN than LPN and OPN. However, we found no statistically significant difference in postoperative complications among our three groups. 
Our study has three main limitations. First, it was a retrospective study with a small sample size. Second, the surgeries were performed by eight surgeons, which may have resulted in different outcomes. Third, performance of the eGFR evaluation at 1 year may have been too early to determine the actual long-term renal function outcomes.

\section{Conclusion}

There was no statistically significant difference in trifecta outcome achievement among patients who underwent OPN, LPN, and RAPN. LPN and RAPN were associated with lower EBL, a lower transfusion rate, and comparable pathologic outcomes in comparison with OPN. Therefore, both LPN and RAPN are feasible and safe techniques for the treatment of small renal masses. Moreover, LPN and RAPN may be utilized for the treatment of large renal masses in the future.

\section{Declarations}

Acknowledgments: The authors thank the staff at the Department of Surgery, Ramathibodi Hospital, Mahidol University for their assistance in this study. The authors also thank Miss Suraida Aeesoa for providing the statistical consultation. Finally, the authors thank Angela Morben, DVM, ELS, from Edanz Group (https://en-author-services.edanz.com/ac), for editing a draft of this manuscript.

Author Contribution statement: Chaichant is the first author of this article. Pocharapong is the corresponding author of this article. Chaichant wrote the main manuscript and designed the study. Pocharapong wrote the main manuscript, designed the study, directed the project, and revised the manuscript. Pokket designed the study and collected data. Premsant, Kittinut, Wit, Wisoot, Charoen, and Wachira collected the data.

Funding: Not applicable.

Potential conflicts of interest: None.

Ethics approval: This study was approved by the Committee on Human Rights Related to Research Involving Human Subjects, Faculty of Medicine Ramathibodi Hospital (MURA2019/1183).

Consenttoparticipate: Informed consent was obtained from all subjects or, if subjects are under 18 , from a parent and/or legal guardian.

Consentforpublication: Not applicable.

Availability of data and material: The datasets used and/or analysed during the current study available from the corresponding author on reasonable request.

Code availability: Not applicable. 


\section{References}

1. Siegel RL, Miller KD, Jemal A (2017) Cancer Statistics, 2017. CA Cancer J Clin 67:7-30.

2. Partin A, Peters C, Kavoussi L, Dmochowski R, Wein A (eds) (2020) Campbell Walsh Wein Urology, 12th edn. Elsevier, Philadelphia.

3. Johnson DC, et al. (2015) Preoperatively misclassified, surgically removed benign renal masses: a systematic review of surgical series and United States population level burden estimate. J Urol 193:30-35.

4. Thompson $\mathrm{RH}$, et al. (2009) Metastatic renal cell carcinoma risk according to tumor size. J Urol 182:41-45.

5. Pierorazio PM, et al. (2016) Distinguishing malignant and benign renal masses with composite models and nomograms: a systematic review and meta-analysis of clinically localized renal masses suspicious for malignancy. Cancer 122:3267-3276.

6. Jayson M, Sanders H (1998) Increased incidence of serendipitously discovered renal cell carcinoma. Urology 51:203-205.

7. Huang WC, et al. (2006) Chronic kidney disease after nephrectomy in patients with renal cortical tumours: a retrospective cohort study. Lancet Oncol 7:735-740.

8. Campbell S, et al. (2017) Renal mass and localized renal cancer: AUA Guideline. J Urol 198:520-529.

9. Mir MC, et al. (2017) Partial nephrectomy versus radical nephrectomy for clinical T1b and T2 renal tumors: a systematic review and meta-analysis of comparative studies. Eur Urol 71:606-617.

10. Huang WC, et al. (2009) Partial nephrectomy versus radical nephrectomy in patients with small renal tumors-is there a difference in mortality and cardiovascular outcomes? J Urol 181:55-61; discussion 61-62.

11. An JY, et al. (2017) Partial vs radical nephrectomy for T1-T2 renal masses in the elderly: comparison of complications, renal function, and oncologic outcomes. Urology 100:151-157.

12. Motzer RJ, et al. (2020) NCCN Guidelines Insights: Kidney Cancer, Version 1.2021. J Natl Compr Canc Netw 18:1160-1170.

13. Porpiglia F, et al. (2016) Partial Nephrectomy in Clinical T1b Renal Tumors: Multicenter Comparative Study of Open, Laparoscopic and Robot-assisted Approach (the RECORd Project). Urology 89:45-51.

14. Pasticier G, et al. (2001) Robotically assisted laparoscopic radical prostatectomy: feasibility study in men. Eur Urol 40:70-74.

15. Yerram NK, et al. (2018) Trifecta outcomes in multifocal tumors: a comparison between robotic and open partial nephrectomy. J Endourol 32:615-620.

16. Mehra K, et al. (2019) Trifecta outcomes in open, laparoscopy or robotic partial nephrectomy: does the surgical approach matter? J Kidney Cancer VHL 6:8-12.

17. Khalifeh A, et al. (2013) Comparative outcomes and assessment of trifecta in 500 robotic and laparoscopic partial nephrectomy cases: a single surgeon experience. J Urol 189:1236-1242. 
18. Zargar $\mathrm{H}$, et al. (2014) Comparison of perioperative outcomes of robot-assisted partial nephrectomy and open partial nephrectomy in patients with a solitary kidney. J Endourol 28:1224-1230.

19. Xia L, et al. (2017) Systematic review and meta-analysis of comparative studies reporting perioperative outcomes of robot-assisted partial nephrectomy versus open partial nephrectomy. $J$ Endourol 31:893-909.

20. Zargar H, et al. (2015) Trifecta and optimal perioperative outcomes of robotic and laparoscopic partial nephrectomy in surgical treatment of small renal masses: a multi-institutional study. BJU Int 116:407-414.

21. Han KS, et al. (2017) Comparison of hand-assisted laparoscopic vs robot-assisted laparoscopic vs open partial nephrectomy in patients with T1 renal masses. J Endourol 31:374-379.

22. Kim JK, et al. (2019) Comparison of robotic and open partial nephrectomy for highly complex renal tumors (RENAL nephrometry score $\geq 10$ ). PLoS One 14:e0210413. 